\title{
Trois ans, cinq ans ou toute la vie?
}

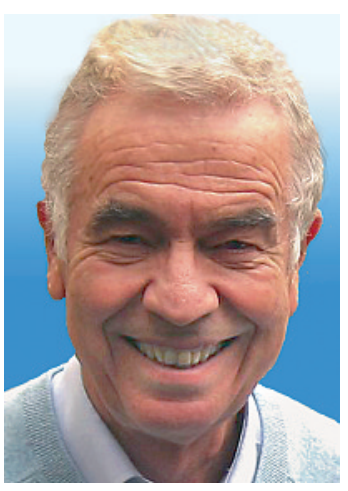

Hans Stalder
Le temps de formation postgraduée pour obtenir le titre de médecine générale/médecine de famille varie dans différents pays: dans certains, elle ne dure que deux ans, plusieurs pays de l'UE ainsi que les Etats-Unis ont opté pour trois et d'autres comme l'Allemagne et le Danemark pour cinq ans. En Suisse, la Société suisse de médecine générale (SSMG) se bat pour garder également cinq ans au point de vouloir même abandonner son titre de spécialiste de médecine générale pour le troquer contre celui de médecine interne, ce qui pourrait lui coûter l'affiliation tant médiatisée à la Wonca. La raison est la libre circulation entre les pays de l'UE et la Suisse; dans l'UE la formation minimum est de trois ans seulement, jugée insuffisant par la SSGM: indigne de fraterniser avec un collègue formé «à bas prix» pendant seulement trois ans!

Est-ce pour autant que les généralistes suisses et allemands sont plus performants que par exemple les anglais ou néerlandais? Pas si sûr! Des études comparatives n'existent que pour la satisfaction des patients, où effectivement, les suisses se trouvent en bonne place. Mais la satisfaction du patient semble plutôt dépendre de l'accessibilité au médecin et du libre choix que de la qualité de la prestation.

Si nous admettons que, au moins dans certains pays, le résultat de la formation courte soit aussi bon que chez nous, comment font-ils pour arriver à produire des généralistes de haut niveau après seulement trois ans? Il y a plusieurs possibilités: La formation prégraduée des étudiants pourrait être plus performante dans le domaine de la médecine de premier recours. Ainsi, les candidats pour la formation en médecine générale y seraient mieux préparés. Ceci est certainement le cas pour les Pays-Bas et le Royaume-Uni, où les départements facultaires de médecine de famille sont très influents et contribuent plus à l'enseignement prégradué en médecine de premier recours que c'est le cas chez nous.

La condition principale pour former d'excellents médecins de famille en seulement trois ans est cependant une définition claire des objectifs de formation et, à part une évaluation adaptée à ces objectifs, surtout une mise à disposition de moyens pédagogiques et financiers adéquats pour les réaliser. Ainsi, aux Pays-Bas et au Royaume-Uni la formation au cabinet est trois à quatre fois plus longue qu'à l’hôpital, les médecins généralistes installés sont bien formés à cet enseignement et les facultés participent à la formation, qui de plus est structurée (aux Pays-Bas sous formes de cours théoriques et de séminaires).

A ceci s'ajoute encore autre chose. Si d'antan la formation était achevée après l'obtention du titre, il est évident qu'aujourd'hui il s'agit d'un processus continu pendant toute la carrière. La formation continue doit automatiquement suivre la formation postgraduée. Comme celle-ci, elle doit être structurée, évaluée (pas nécessairement par des examens obligatoires) et surtout reconnue, ce qui n'est guère le cas chez nous.

Bien qu'il soit évident qu'il faut un minimum de temps, ce n'est donc pas la durée en soi qui compte pour la formation - de toute façon elle durera toute la vie - mais la qualité: définir clairement les objectifs, mettre en œuvre de moyens adéquats et organiser une évaluation adaptée, comme toujours dans tout cycle pédagogique. Cette formation de médecine générale doit commencer le premier jour des études et finir seulement en fin de carrière avec un intermezzo de trois ans de «formation postgraduée». Irréalisable en Suisse? Si c'est possible dans d'autres pays, pourquoi pas chez nous? Pourquoi ne pas au moins y réfléchir (par exemple: est-ce que l'année à choix est indispensable, correspond-elle aux objectifs de formation - devenir un bon généraliste? Ça ferait déjà un an de gagné ...)?

Voici une réflexion intéressante à faire pour les nouveaux Instituts de médecine générale: qu'elles seraient les conditions de former en Suisse des excellents généralistes en seulement trois ans? Comment le réaliser? Comment surmonter les obstacles? Au moins, cela permettrait aux généralistes de ne pas devenir «spécialistes» et de garder le beau titre, devenu européen, de médecine générale/médecine de famille ...

Hans Stalder*

PS: Evidemment, les mêmes arguments peuvent être appliqués à d'autres formations de médecins de premier recours: aux Etats-Unis, par exemple, la formation postgraduée des internistes et des pédiatres ne dure également que trois ans ... 